\title{
Diversity of ethnobotanically significant angiospermic weeds in Siang Belt of Arunachal Himalayan Region in India
}

\author{
Momang Taram ${ }^{1}$, Rubu Rinyo ${ }^{1}$, Lemmem Gammi ${ }^{1}$, Kenba Yanggi ${ }^{1}$, Atek \\ Nangkar ${ }^{1}$, Pallabi K. Hui ${ }^{2}$, Sanjay Jambhulkar ${ }^{3}$ and Hui Tag,4 \\ ${ }^{1}$ Plant Systematics and Ethnobotanical Research Laboratory, Department of Botany, Rajiv Gandhi \\ University, Rono Hills, Doimukh- 791112, Arunachal Pradesh, India \\ ${ }^{2}$ Department of Biotechnology, National Institute of Technology - Arunachal Pradesh, Yupia-791112, \\ Papum Pare, Arunachal Pradesh, India \\ ${ }^{3}$ Nuclear Agriculture \& Biotechnology Division, Bhabha Atomic Research Centre, Trombay, Mumbai- \\ 400085, India \\ ${ }^{4}$ Corresponding author; E-mail: huitag2008rgu@gmail.com
}

[Received 11.11.2019; Revised \& accepted 22.12.2019; Published 31.12.2019]

\begin{abstract}
With objective to investigate the diversity of ethnobotanically significant angiospermic weeds used among the Adi community in the Siang belt of Arunachal Pradesh in their traditional biocultural landscape has recorded a total of 87 weed species. Many of these are economic significant and growing luxuriantly on the roadside, wasteland, jhum land and valleys. Their uses include food, human herbal medicines, veterinary medicines, fish poisoning agent, and use in local customary rituals practices.
\end{abstract}

Key words: Ethnobotany; Angiosperm Weeds; Utilization, Siang Belt; Arunachal Himalaya

\section{INTRODUCTION}

Etymologically, "weed" derives from the old English word for "grass" or "herb," but during the middle ages the meaning has been changed to indicate an undesirable plant that grows where it is not wanted, especially along the agricultural plots (Rahman 2013). Baker (1965) deûnes a weed as a plant in any specify geographical area, its populations grow entirely or predominantly in situations markedly in disturbed environments, fast growing and are not always herbaceous (Zimdahl 1992). The notion of weeds as unnecessary plants was originated when man started to intentionally growing plants for food. They invade natural vegetation, usually adversely affecting native biodiversity or ecosystem functioning as well as competing with crop plants for common resources such as water, mineral nutrients, space and light (Heywood 1995; Rahman et al. 2011; Maroyi 2013). Some weeds produce chemical substances which are toxic to crop plants but not all the weeds are unwanted. In the rangeland areas, many annual grasses considered as weeds in crops are useful as animal feed. They also serve as food and shelter for wildlife, reduce soil erosion as good soil binders, and are useful for food and medicinal purposes (Neogi et al. 1989).

India has a wide range of agro-climates and soil types with highly diverse agriculture and farming systems beset with different types of weeds, which are very common, dominant and wide spread in the crop fields (Dhole et al. 2009; Rao \& Chauhan 2015). Ethnobotanically important rare, endangered primary forest plant species can be replaced by invasive and exotic weeds, which have higher potential to survive and reproduce in stressed environment (Neogi et al. 1989). In the context of India and Northeast India, no systematic documentation 
on weed flora are available to date. Hence, this paper aims to discuss on the diversity, distribution, ecology and economic importance of the ethnobotanically significant weed flora recorded from Adi inhabited Siang Belt of Arunachal Pradesh based on the ethnobotanical survey conducted during $2017-2019$ and it is expected that this baseline taxonomic data would be useful for the management of weeds flora in Eastern Himalayan region in more productive and sustainable manner.

\section{MATERIALS \& METHODS}

\section{Study site and local cultural group}

Ethnobotanical field study was conducted from 2017-2019 in the 8 villages of Siang districts, namely, Geku, Komkar, Yingkyong, Pasighat, Mebo, Riew, Boleng and Kerang which falls under tropical and subtropical climatic zones of East Siang, Upper Siang and Siang districts of Arunachal Pradesh, India. These districts are predominantly inhabited by the indigenous Adi tribal community living in the foot hills and the Bank of Mighty Siang River in the Eastern Himalayan region of India. The Adi people of Siang area still practices their ageold traditional faith and belief system called Donyi-Polo and practice terrace and jhum agriculture for sustenance of livelihood (Tag et al. 2008).

\section{Ethnobotanical documentation of weeds flora}

A total of 100 respondents from 50 household covering 8 villages of Adi localities were interviewed following the method suggested by Martin (2008). Prior Inform Consent (PIC) was taken from the potential informants before start of the work.Ethnobotanical uses of weed flora encountered were recorded in the structured questionnaire format and field notebook, and live photos of each species were captured. The voucher specimens were collected, dried, mounted, labelled and preserved following the method suggested by Jain and Rao (1977). Each plant was identified using standard regional and national floras, including Materials for the flora of Arunachal Pradesh (Hajra et al. 1996; Giri et al. 2008; Chowdhery et al. 2009); The Flora of British India (Hooker 1872 - 1897) and e-flora of India. The accepted scientific names were verified in the website www.theplantlist.org hosted by Royal Botanic Garden Kew UK and Missouri Botanical Garden, St. Louis, and Plants of World Online (POWO) hosted by RBG Kew. Voucher specimens were deposited in the Herbarium of Arunachal University (HAU), Department of Botany, Rajiv Gandhi University for future consultation.

\section{RESULT AND DISCUSSION}

\section{Weed species diversity and traditional uses}

Present investigation has recognized 87 weed species falling under 64 genera and 31 families which belong to Angiosperms which is presented in Table 1. Majority of the species (44\%) reported are having local food value while $27 \%$ species are used as medicine, $11 \%$ species are used for other purposes, $10 \%$ species are used for fodders and only $8 \%$ of the total species reported are used for traditional customary rituals. Of the total 87 weed species reported, 42 species are exclusively used as food supplements. These species are widespread in distribution right from road side to invading jhum field, kitchen garden and wasteland in the Eastern Himalaya including the Adi belt of Arunachal Pradesh. The edible and ethnobotanically significant weed species reported from current study sites are: Gynura cosimbua, Fagopyrum esculentum, Houttuynia cordata, Gonostegia hirta, Solanum americanum, Solanum torvum, etc. commonly domesticated in the kitchen garden and found in wild in jhum land. 

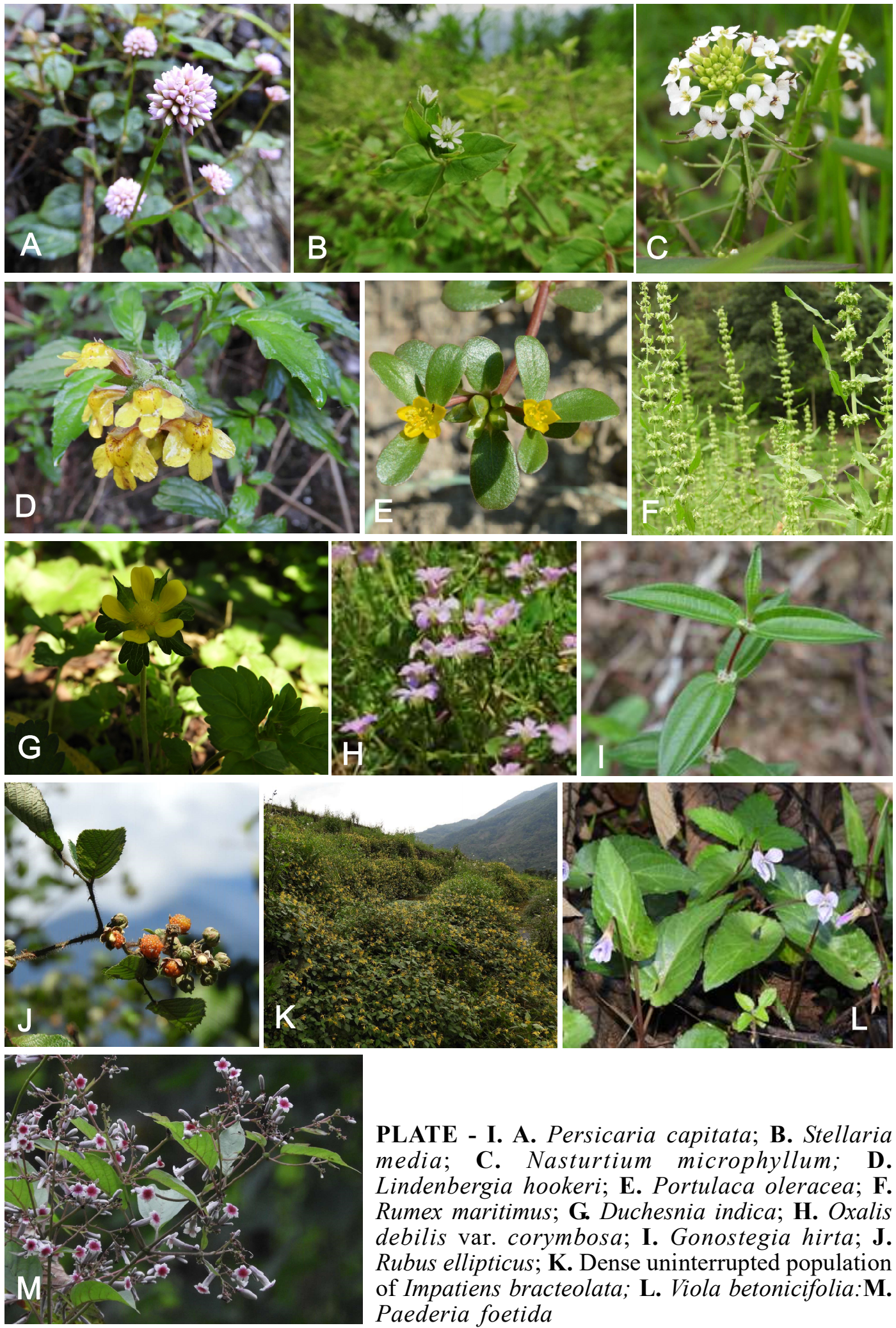

PLATE - I. A. Persicaria capitata; B. Stellaria media; C. Nasturtium microphyllum; D. Lindenbergia hookeri; E. Portulaca oleracea; F. Rumex maritimus; G. Duchesnia indica; H. Oxalis debilis var. corymbosa; I. Gonostegia hirta; J. Rubus ellipticus; K. Dense uninterrupted population of Impatiens bracteolata; L. Viola betonicifolia:M. Paederia foetida 
Table -1. Checklist of ethhnobotanically significant angiospermic weeds in the Siang belt of Arunachal Himalayan Region

[Abbreviation used: $\mathbf{F}=$ Food; $\mathbf{F D}=$ Fodder $; \mathbf{F H}=$ Fishing; $\mathbf{R B C}=$ Rituals beliefs and customs; $\mathbf{V E T}$ $=$ Veterinary $; \mathbf{O}=$ Others; $\mathbf{T U}=$ Traditional uses $]$

\begin{tabular}{|c|c|c|c|c|c|}
\hline $\begin{array}{l}\text { Botanical name [Family]; } \\
\text { Voucher specimen }\end{array}$ & Adi Names & $\mathbf{T} \mathbf{U}$ & Mode of use & $\begin{array}{l}\text { Endemism } \\
\text { range }\end{array}$ & $\begin{array}{l}\text { Marke- } \\
\text { tability }\end{array}$ \\
\hline $\begin{array}{l}\text { Actephila excelsa (Dalzell) Mull. } \\
\text { Arg. [Phyllanthaceae]; LG-134 }\end{array}$ & Kamtar-oying & $\mathrm{F}$ & $\begin{array}{l}\text { Tender shoots taken as } \\
\text { vegetable }\end{array}$ & - & Yes \\
\hline $\begin{array}{l}\text { Ageratum conyzoides (L.) L. } \\
\text { [Asteraceae]; MT-906 }\end{array}$ & $\begin{array}{l}\text { Namsing eeing/ } \\
\text { Migom Dumpu/ } \\
\text { Pakkung eing/ } \\
\text { Bongar/Yabum }\end{array}$ & $\begin{array}{l}\mathrm{M} ; \\
\mathrm{RCB}\end{array}$ & $\begin{array}{l}\text { Leaf paste applied on cuts } \\
\text { and bruises as haemostat; } \\
\text { leaf juice taken orally for } \\
\text { headache and shivering; } \\
\text { Facing of inflorescence } \\
\text { direction signify dusk/ } \\
\text { dawn }\end{array}$ & $\begin{array}{l}\text { Tropical } \\
\text { America and } \\
\text { Mexico }\end{array}$ & No \\
\hline $\begin{array}{l}\text { Ageratum houstonianum Mill. } \\
\text { [Asteraceae]; MT-908 }\end{array}$ & $\begin{array}{l}\text { Namsing eeing/ } \\
\text { Migom Dumpu }\end{array}$ & $\begin{array}{l}\mathrm{M} ; \\
\mathrm{RCB}\end{array}$ & $\begin{array}{l}\text { Leaf paste used as } \\
\text { haemostat on cuts and } \\
\text { bruises; movement of } \\
\text { inflorescence direction } \\
\text { signifies dusk / dawn }\end{array}$ & $\begin{array}{l}\text { Central } \\
\text { America, } \\
\text { Mexico }\end{array}$ & No \\
\hline $\begin{array}{l}\text { Amaranthus spinosus L. } \\
\text { [Amaranthaceae]; GM-142 }\end{array}$ & $\begin{array}{l}\text { Tapi-Pilee / } \\
\text { Gubor }\end{array}$ & $\mathrm{F}$ & $\begin{array}{l}\text { Tender shoots taken as } \\
\text { vegetable }\end{array}$ & $\begin{array}{l}\text { Trop. America, } \\
\text { Mexico }\end{array}$ & Yes \\
\hline $\begin{array}{l}\text { Amaranthus viridis L. } \\
\text { [Amaranthaceae]; GM-143 }\end{array}$ & $\begin{array}{l}\text { Tapi-pilee/ } \\
\text { Gubor }\end{array}$ & $\mathrm{F}$ & $\begin{array}{l}\text { Tender shoots taken as } \\
\text { vegetable }\end{array}$ & $\begin{array}{l}\text { Trop. America, } \\
\text { Mexico }\end{array}$ & Yes \\
\hline $\begin{array}{l}\text { Ardisia solanacea } \text { Roxb. } \\
\text { [Primulaceae]; LG-122 }\end{array}$ & Go-yakpin & $\mathrm{F}$ & $\begin{array}{l}\text { Tender shoots taken as } \\
\text { vegetable }\end{array}$ & $\begin{array}{l}\text { Pakistan, India, } \\
\text { Sri Lanka, SE } \\
\text { Asia, W China }\end{array}$ & No \\
\hline $\begin{array}{l}\text { Artemisia indica Willd. } \\
\text { [Asteraceae]; LG- } 116\end{array}$ & $\begin{array}{l}\text { Eetki-Daali/fagi } \\
\text { Rete/Laglin }\end{array}$ & $\begin{array}{l}\mathrm{M} ; \\
\mathrm{RBC}\end{array}$ & $\begin{array}{l}\text { Leaves paste taken orally } \\
\text { stomach disorder; twigs } \\
\text { used in funerals }\end{array}$ & $\begin{array}{l}\text { East Asia, } \\
\text { China, Japan, } \\
\text { India }\end{array}$ & No \\
\hline $\begin{array}{l}\text { Bidens pilosa L. [Asteraceae]; } \\
\text { GM-145 }\end{array}$ & $\begin{array}{l}\text { Tasso-Lepyo/ } \\
\text { Ejar }\end{array}$ & $\mathrm{F}$ & $\begin{array}{l}\text { Tender shoots taken as } \\
\text { vegetable }\end{array}$ & $\begin{array}{l}\text { America, } \\
\text { Eurasia, Africa, } \\
\text { Australia, } \\
\text { Pacific Island }\end{array}$ & Yes \\
\hline $\begin{array}{l}\text { Blumea balsamifera DC. } \\
\text { [Asteraceae]; LG-119 }\end{array}$ & $\begin{array}{l}\text { Eyok aain/Mine } \\
\text { geyin }\end{array}$ & $\mathrm{M}$ & $\begin{array}{l}\text { Crushed leaves mixed } \\
\text { with Mithun dung and } \\
\text { freshly collected stream- } \\
\text { water is applied on the } \\
\text { forehead to treat malaria }\end{array}$ & $\begin{array}{l}\text { Asia, China, } \\
\text { Indo-China, } \\
\text { Malaysia }\end{array}$ & No \\
\hline $\begin{array}{l}\text { Brachiaria eruciformis (Sm.) } \\
\text { Griseb. [Poaceae]; MT-932 }\end{array}$ & Taami & FD & Fodder for cattle & $\begin{array}{l}\text { Mediterranean } \\
\text { to Indo-China, } \\
\text { Africa }\end{array}$ & No \\
\hline $\begin{array}{l}\text { Brachiaria ramosa (L.) Stapf } \\
\text { [Poaceae]; MT-937 }\end{array}$ & $\begin{array}{l}\text { Taami/Tarum } \\
\text { majit }\end{array}$ & FD & Fodder for cattle & Africa, Asia & No \\
\hline $\begin{array}{l}\text { Brachiaria reptans (L.) C.A. } \\
\text { Gardener \& C.E.Hubb. } \\
\text { [Poaceae]; MT-933 }\end{array}$ & Taami & FD & Fodder for cattle & $\begin{array}{l}\text { Arab peninsula, } \\
\text { Afghanistan to } \\
\text { Pacific }\end{array}$ & No \\
\hline $\begin{array}{l}\text { Brachystemma calycinum } \\
\text { D.Don [Caryophyllaceae]; MT- } \\
912\end{array}$ & Okin-Parin & $\mathrm{F} ; \mathrm{M}$ & $\begin{array}{l}\text { Leaves are warmed after } \\
\text { packing with Phrynium } \\
\text { pubinerve leaves and then } \\
\text { applied to cure cracked } \\
\text { sole }\end{array}$ & $\begin{array}{l}\text { SW China, } \\
\text { Nepal }\end{array}$ & No \\
\hline $\begin{array}{l}\text { Bryophyllum pinnatum (Lam.) } \\
\text { Oken [Crassulaceae]; MT-909 }\end{array}$ & $\begin{array}{l}\text { Nevi nelaum, } \\
\text { Eme kuserang }\end{array}$ & $\mathrm{M}$ & $\begin{array}{l}\text { Plant extract applied on } \\
\text { affected body part to cure } \\
\text { burn and inflammation; } \\
\text { leaves eaten raw to clean } \\
\text { stomach }\end{array}$ & $\begin{array}{l}\text { S Africa, } \\
\text { Madagascar, } \\
\text { Asia }\end{array}$ & No \\
\hline $\begin{array}{l}\text { Cardamine hirsuta L. } \\
\text { [Brassicaceae]; MT-917 }\end{array}$ & $\begin{array}{l}\text { Oram-Petsik/ } \\
\text { Loram pattu }\end{array}$ & $\mathrm{F}$ & Whole plants edible & $\begin{array}{l}\text { Europe, } \mathrm{N} \\
\text { Africa }\end{array}$ & No \\
\hline $\begin{array}{l}\text { Carex baccans Nees } \\
\text { [Cyperaceae]; LG-120 }\end{array}$ & $\begin{array}{l}\text { Gemin-Taabeng/ } \\
\text { Tapok/ Tabeng }\end{array}$ & RBC & $\begin{array}{l}\text { They belief that Carex } \\
\text { baccans and Saccharum } \\
\text { aruninaceum came from } \\
\text { the same ancestor so both } \\
\text { are used together in } \\
\text { funeral rituals }\end{array}$ & $\begin{array}{l}\text { India, Sri Lanka, } \\
\text { China }\end{array}$ & No \\
\hline
\end{tabular}




\begin{tabular}{|c|c|c|c|c|c|}
\hline $\begin{array}{l}\text { Botanical name [Family]; } \\
\text { Voucher specimen }\end{array}$ & Adi Names & T U & Mode of use & $\begin{array}{l}\text { Endemism } \\
\text { range }\end{array}$ & $\begin{array}{l}\text { Marke- } \\
\text { tability }\end{array}$ \\
\hline $\begin{array}{l}\text { Centella asiatica (L.) Urb. } \\
\text { [Apiaceae]; LG-139 }\end{array}$ & $\begin{array}{l}\text { Kiiling Kiipum, } \\
\text { Golgi-Sipum, } \\
\text { Dolgi/Jorgang } \\
\text { taek }\end{array}$ & $\mathrm{M}$ & $\begin{array}{l}\text { Paste of whole plants } \\
\text { taken orally to treat } \\
\text { gastritis }\end{array}$ & Asia & Yes \\
\hline $\begin{array}{l}\text { Chenopodium album L. } \\
\text { [Amarantaceae]; LG-129 }\end{array}$ & Jili-Mili/ Taye & $\mathrm{F}$ & $\begin{array}{l}\text { Tender shoots taken as } \\
\text { vegetable }\end{array}$ & $\begin{array}{l}\text { Europe, Eurasia } \\
\text { to India }\end{array}$ & Yes \\
\hline $\begin{array}{l}\text { Chenopodium giganteum D.Don } \\
\text { [Amaranthaceae]; LG- } 103\end{array}$ & Amateng & $\mathrm{F}$ & $\begin{array}{l}\text { Tender shoots taken as } \\
\text { vegetable }\end{array}$ & $\begin{array}{l}\text { Himalayas to } \\
\text { Korea }\end{array}$ & Yes \\
\hline $\begin{array}{l}\text { Chromolaena odorata (L.) } \\
\text { R.M.King \& H.Rob. [Asteraceae]; } \\
\text { LG-126 }\end{array}$ & Ingkir & $\mathrm{M}$ & $\begin{array}{l}\text { Leaf -paste applied on } \\
\text { cuts for blood clotting }\end{array}$ & America & No \\
\hline $\begin{array}{l}\text { Crassocephalum crepidioides } \\
\text { (Benth.) S.Moore [Asteraceae]; } \\
\text { LG- } 115\end{array}$ & $\begin{array}{l}\text { Eeli/Jogen/ } \\
\text { Telimbabo/ } \\
\text { Gendeh }\end{array}$ & $\mathrm{F}$ & $\begin{array}{l}\text { Tender shoots taken as } \\
\text { vegetable }\end{array}$ & Africa & Yes \\
\hline $\begin{array}{l}\text { Cynodon dactylon (L.) Pers. } \\
\text { [Poaceae]; MT-936 }\end{array}$ & $\begin{array}{l}\text { Taami / Tarum } \\
\text { majut }\end{array}$ & FD & Fodder for cattle & $\begin{array}{l}\text { E Africa, } \\
\text { Australia }\end{array}$ & No \\
\hline $\begin{array}{l}\text { Dinebra retroflexa (Vahl) Panz. } \\
\text { [Poaceae]; MT-934 }\end{array}$ & Taami & FD & Fodder for cattle & Africa & No \\
\hline $\begin{array}{l}\text { Drymaria cordata (L.) Willd. ex } \\
\text { Schult. [Caryophyllaceae]; MT- } \\
929\end{array}$ & $\begin{array}{l}\text { Pipi, Perok } \\
\text { taiter/Kumting } \\
\text { karlor }\end{array}$ & $\mathrm{M}$ & $\begin{array}{l}\text { Paste of whole plant used } \\
\text { locally to cure ringworm }\end{array}$ & $\begin{array}{l}\text { Mexico, West } \\
\text { Indies, S Africa, } \\
\text { C \& S America }\end{array}$ & No \\
\hline $\begin{array}{l}\text { Duchesnea indica (Jacks.) Focke } \\
\text { [ Rosaceae]; LG } 118\end{array}$ & $\begin{array}{l}\text { Eki-Tangkin/ } \\
\text { Pamik taang }\end{array}$ & $\mathrm{F}$ & Ripe fruits edible (watery) & $\begin{array}{l}\text { E \& S Asia, } \\
\text { Afghanistan to } \\
\text { Russian Far } \\
\text { East, Malaysia }\end{array}$ & No \\
\hline $\begin{array}{l}\text { Eclipta prostrata (L.) L. } \\
\text { [Asteraceae]; LG-137 }\end{array}$ & $\begin{array}{l}\text { Keharaj/Donyi } \\
\text { Hangkang }\end{array}$ & $\mathrm{M}$ & $\begin{array}{l}\text { Plant decoction taken } \\
\text { orally to cure dysentery }\end{array}$ & $\begin{array}{l}\text { America, India, } \\
\text { Nepal, China, } \\
\text { Thailand, Brazil }\end{array}$ & No \\
\hline $\begin{array}{l}\text { Eragrostis minor Host } \\
\text { [Poaceae]; MT-935 }\end{array}$ & Taami & FD & Fodder for cattle & Eurasia, Africa & No \\
\hline $\begin{array}{l}\text { Erigeron canadensis } \mathrm{L} . \\
\text { [Asteraceae]; LG-127 }\end{array}$ & $\begin{array}{l}\text { Ingkobodong/ } \\
\text { Eedong }\end{array}$ & $\mathrm{F}$ & $\begin{array}{l}\text { Tender shoots taken as } \\
\text { vegetable }\end{array}$ & N \& C America & No \\
\hline $\begin{array}{l}\text { Euphorbia hirta L. } \\
\text { [Euphorbiaceae]; MT-902 }\end{array}$ & Korek oying & $\begin{array}{l}\mathrm{FD} \\
\mathrm{M}\end{array}$ & $\begin{array}{l}\text { Whole plant used as pig- } \\
\text { fodder; leaves taken } \\
\text { orally against flies }\end{array}$ & Trop. America & No \\
\hline $\begin{array}{l}\text { Fagopyrum esculentum Moench } \\
\text { [Polygonaceae] }\end{array}$ & Okung, Lompuk & $\mathrm{F}$ & $\begin{array}{l}\text { Tender shoot taken as } \\
\text { vegetable }\end{array}$ & $\begin{array}{l}\text { Native to C \& N } \\
\text { Asia }\end{array}$ & Yes \\
\hline $\begin{array}{l}\text { Gnaphalium polycaulon Pers. } \\
\text { [Asteraceae]; MT- } 923\end{array}$ & Paaput & $\mathrm{F}$ & Leaves edible & $\begin{array}{l}\text { Mesoamerica, S } \\
\text { America, West } \\
\text { Indies }\end{array}$ & No \\
\hline $\begin{array}{l}\text { Gynura cusimbua (D.Don) } \\
\text { S.Moore [Asteraceae]; MT-910 }\end{array}$ & Ogen & $\mathrm{F}$ & Leaves edible & $\begin{array}{l}\text { China, Tibet, } \\
\text { India, Myanmar, } \\
\text { Nepal, Thailand, } \\
\text { Bangladesh, } \\
\text { Bhutan }\end{array}$ & Yes \\
\hline $\begin{array}{l}\text { Houttuynia cordata Thumb. } \\
\text { [Saururaceae]; MT- } 930\end{array}$ & Roram & F; M & $\begin{array}{l}\text { Whole plant made into } \\
\text { chutney; use in } \\
\text { stomachache }\end{array}$ & SE Asia & Yes \\
\hline $\begin{array}{l}\text { Hydrocotyle javanica Thunb. } \\
\text { [Araliaceae]; LG-140 }\end{array}$ & Kiling-Kiipum & $\mathrm{O}$ & $\begin{array}{l}\text { Whole plants used to } \\
\text { stupefy fishes }\end{array}$ & $\begin{array}{l}\text { NE India, SE } \\
\text { Asia, Fiji }\end{array}$ & No \\
\hline $\begin{array}{l}\text { Impatiens bracteolata Hook.f. } \\
\text { [Balsaminaceae]; MT-907 }\end{array}$ & Nanor-Tangkor & $\mathrm{F}$ & Tender shoots edible & $\begin{array}{l}\text { E. Himalaya to } \\
\text { Myanmar. }\end{array}$ & No \\
\hline $\begin{array}{l}\text { Laphangium affine (D.Don) } \\
\text { Tzvelev [Asteraceae]; MT-922 }\end{array}$ & Paaput & $\mathrm{F}$ & Leaves edible & $\begin{array}{l}\text { Temperate } \\
\text { region of China, } \\
\text { Korea, Japan, } \\
\text { Taiwan, high } \\
\text { altitude tropical } \\
\text { region of India, } \\
\text { Nepal, Thailand }\end{array}$ & \\
\hline
\end{tabular}


Momang Taram et al. 341

\begin{tabular}{|c|c|c|c|c|c|}
\hline $\begin{array}{l}\text { Botanical name [Family]; } \\
\text { Voucher specimen }\end{array}$ & Adi Names & $\mathbf{T} \mathbf{U}$ & Mode of use & $\begin{array}{l}\text { Endemism } \\
\text { range }\end{array}$ & $\begin{array}{l}\text { Marke- } \\
\text { tability }\end{array}$ \\
\hline $\begin{array}{l}\text { Leucas aspera (Willd.) Link } \\
\text { [Lamiaceae]; LG-117 }\end{array}$ & Eki sipak & $\mathrm{M}$ & $\begin{array}{l}\text { Leaf-paste used externally } \\
\text { on nose to cure sinus } \\
\text { problems }\end{array}$ & $\begin{array}{l}\text { India, Mauritius, } \\
\text { Philippines, Java }\end{array}$ & No \\
\hline $\begin{array}{l}\text { Lindenbergia hookeri } \\
\text { C.B.Clarke ex Hook.f. } \\
\text { [Orobanchaceae]; MT-925 }\end{array}$ & Pepit namdung & $\bar{F}$ & Flowers are edible (sour) & E. Himalaya & No \\
\hline $\begin{array}{l}\text { Melastoma malabathricum L. } \\
\text { [Melastomataceae]; LG-136 }\end{array}$ & Kasii Rai/ Joger & $\begin{array}{l}\mathrm{F} \\
\mathrm{RBC}\end{array}$ & $\begin{array}{l}\text { Fruits edible; its blooming } \\
\text { signals that the high time } \\
\text { for paddy-seeds } \\
\text { broadcasting }\end{array}$ & $\begin{array}{l}\text { Indo-Malaya, } \\
\text { Japan, Australia }\end{array}$ & No \\
\hline $\begin{array}{l}\text { Mikania micrantha Kunth } \\
\text { [Asteraceae]; LG-114 }\end{array}$ & $\begin{array}{l}\text { Eeli/Eeing } \\
\text { mamang }\end{array}$ & $\mathrm{M}$ & $\begin{array}{l}\text { Leave used to cure } \\
\text { stomachache and } \\
\text { dysentery }\end{array}$ & $\begin{array}{l}\text { Subtrop. N, C \& } \\
\text { S America }\end{array}$ & No \\
\hline $\begin{array}{l}\text { Murdannia nudiflora (L.) } \\
\text { Brenan [Commelinaceae]; LG- } \\
124\end{array}$ & $\begin{array}{l}\text { Hodog/Golgi/ } \\
\text { Gorgi roksok }\end{array}$ & $\mathrm{RBC}$ & $\begin{array}{l}\text { flowering of the plant } \\
\text { signals villagers that it is } \\
\text { time to broadcast paddy- } \\
\text { seeds; the plant is grown in } \\
\text { field as demarcation line }\end{array}$ & $\begin{array}{l}\text { Trop. \& } \\
\text { subtrop. Asia }\end{array}$ & No \\
\hline $\begin{array}{l}\text { Nasturtium microphyllum } \\
\text { (Boenn.) Rchb. [Brassicaceae]; } \\
\text { MT-919 }\end{array}$ & $\begin{array}{l}\text { Orgyam/Patang } \\
\text { oying }\end{array}$ & $\mathrm{F}$ & Leaves edible & $\begin{array}{l}\text { Middle-east, } \\
\text { parts of } \mathrm{N} \\
\text { Africa, Europe }\end{array}$ & Yes \\
\hline $\begin{array}{l}\text { Oenanthe javanica (Blume) DC. } \\
\text { [Apiaceae]; LG-101 }\end{array}$ & - & $\bar{F}$ & $\begin{array}{l}\text { Tender shoots served as } \\
\text { vegetable }\end{array}$ & $\begin{array}{l}\text { Temp. Asia, } \\
\text { Trop. Asia, } \\
\text { Queensland, } \\
\text { Australia }\end{array}$ & Yes \\
\hline $\begin{array}{l}\text { Oxalis corniculata L. } \\
\text { [Oxalidaceae]; MT-926 }\end{array}$ & $\begin{array}{l}\text { Piiag-Hiyu, Piak } \\
\text { Iip }\end{array}$ & $\mathrm{M}$ & $\begin{array}{l}\text { Fruit-juice used to cure } \\
\text { eye infection }\end{array}$ & S America & No \\
\hline $\begin{array}{l}\text { Oxalis debilis var. corymbosa } \\
\text { (DC.) Lourteig [Oxalidaceae]; } \\
\text { MT-927 }\end{array}$ & $\begin{array}{l}\text { Piiag-Hiyub, } \\
\text { Piak Iip }\end{array}$ & $\mathrm{F}$ & $\begin{array}{l}\text { Flowers and rhizome } \\
\text { edible }\end{array}$ & $\begin{array}{l}\text { C America to } \\
\text { Guyana, } \\
\text { Paraguay }\end{array}$ & No \\
\hline $\begin{array}{l}\text { Paederia foetida L. [Rubiaceae] } \\
\text { GM-146 }\end{array}$ & $\begin{array}{l}\text { Yape Taari/Riki } \\
\text { Ringkom/Yepe } \\
\text { ribung }\end{array}$ & $\mathrm{M}$ & $\begin{array}{l}\text { Leaves taken orally to } \\
\text { cure gastritis }\end{array}$ & $\begin{array}{l}\text { Temp. \& trop. } \\
\text { Asia }\end{array}$ & No \\
\hline $\begin{array}{l}\text { Persicaria barbata (L.) H.Hara } \\
\text { [Polygonaceae]; LG-107 }\end{array}$ & Diko-Taamu & $\mathrm{O}$ & $\begin{array}{l}\text { Crushed plants used to } \\
\text { stupefy fish }\end{array}$ & $\begin{array}{l}\text { India, Myanmar, } \\
\text { China, Pegu }\end{array}$ & No \\
\hline $\begin{array}{l}\text { Persicaria capitata (Buch.-Ham. } \\
\text { ex D.Don) H.Gross } \\
\text { [Polygonaceae]; LG-104 }\end{array}$ & $\begin{array}{l}\text { Babing-kaling, } \\
\text { Mijing kaling, } \\
\text { Tasum momi/ } \\
\text { Kibu nanung }\end{array}$ & $\mathrm{F}$ & Ripe fruits edible & Asia & Yes \\
\hline $\begin{array}{l}\text { Persicaria chinensis }(\mathrm{L} .) \mathrm{H} . \\
\text { Gross [Polygonaceae]; LG-102 }\end{array}$ & $\begin{array}{l}\text { Babing-kaling, } \\
\text { Mijing kaling, } \\
\text { Tasum momi/ } \\
\text { Kibu nanung }\end{array}$ & $\mathrm{O}$ & $\begin{array}{l}\text { Crushed plants used to } \\
\text { wash hands in the field; } \\
\text { plant-paste applied on } \\
\text { cuts and wound }\end{array}$ & $\begin{array}{l}\text { Native to trop. } \\
\text { to temp. Asia }\end{array}$ & No \\
\hline $\begin{array}{l}\text { Persicaria hydropiper (L.) } \\
\text { Delarbre [Polygonaceae]; LG- } \\
108\end{array}$ & $\begin{array}{l}\text { Diko-Taamu/ } \\
\text { eeing killing }\end{array}$ & $\mathrm{O}$ & $\begin{array}{l}\text { Crushed leaves used to } \\
\text { stupefy fishes }\end{array}$ & $\begin{array}{l}\text { Australia, New } \\
\text { Zealand, temp. } \\
\text { Asia, Europe, N } \\
\text { America, Africa }\end{array}$ & No \\
\hline $\begin{array}{l}\text { Persicaria nepalenseis (Meisn.) } \\
\text { H.Gross [Polygonaceae]; LG- } \\
105\end{array}$ & $\begin{array}{l}\text { Babing-kaling, } \\
\text { Mijing kaling, } \\
\text { Tasum momi/ } \\
\text { Rungkung }\end{array}$ & $\mathrm{O}$ & $\begin{array}{l}\text { Crushed plant used to } \\
\text { wash hands in the field }\end{array}$ & $\begin{array}{l}\text { E Africa, } \\
\text { including } \\
\text { Madagascar, } \\
\text { parts of Asia }\end{array}$ & No \\
\hline $\begin{array}{l}\text { Phyllanthus amarus Schumach. } \\
\text { \&Thonn. [Phyllanthaceae]; MT- } \\
901\end{array}$ & Kobelang/Eeyup & $\mathrm{M}$ & $\begin{array}{l}\text { Plant-paste taken orally } \\
\text { against jaundice }\end{array}$ & $\begin{array}{l}\text { Mexico to Trop. } \\
\text { America. }\end{array}$ & No \\
\hline $\begin{array}{l}\text { Physalis minima } \mathrm{L} . \\
\text { [Solanaceae]; LG } 131\end{array}$ & Jojing belang & $\mathrm{F}$ & Ripe fruits edible & $\begin{array}{l}\text { Trop. \& Subtrop. } \\
\text { America }\end{array}$ & No \\
\hline $\begin{array}{l}\text { Pilea insolens Wedd. } \\
.[\text { Urticaceae }] ; \text { MT-940 }\end{array}$ & Tango-Lisak & $\mathrm{F}$ & $\begin{array}{l}\text { Leaves used in } \\
\text { fermentation of Perilla } \\
\text { ocymoides seeds }\end{array}$ & $\begin{array}{l}\text { S-E Xizang, } \\
\text { China, Bhutan, } \\
\text { N India, Nepal }\end{array}$ & No \\
\hline $\begin{array}{l}\text { Pilea umbrosa Wedd. ex Blume } \\
\text { [Urticaceae]; MT-915 }\end{array}$ & Oko-Robo & $\mathrm{F}$ & Tender shoots edible & $\begin{array}{l}\text { Pakistan, China, } \\
\text { N Vietnam }\end{array}$ & No \\
\hline
\end{tabular}




\begin{tabular}{|c|c|c|c|c|c|}
\hline $\begin{array}{l}\text { Botanical name [Family]; } \\
\text { Voucher specimen }\end{array}$ & Adi Names & T U & Mode of use & $\begin{array}{l}\text { Endemism } \\
\text { range }\end{array}$ & $\begin{array}{l}\text { Marke- } \\
\text { tability }\end{array}$ \\
\hline $\begin{array}{l}\text { Plantago asiatica } \mathrm{L} . \\
\text { [Plantaginaceae }] ; \text { LG-111 }\end{array}$ & $\begin{array}{l}\text { Donyi-Borkor } \\
\text { Donyi hankeng, } \\
\text { Donyi sokang }\end{array}$ & $\mathrm{F}$ & Leaves edible & E China & No \\
\hline $\begin{array}{l}\text { Polygonum molle D.Don } \\
\text { [Polygonaceae]; LG-138 }\end{array}$ & Kiibu-Nanung & $\mathrm{F}$ & Tender shoots edible & $\begin{array}{l}\text { Indian } \\
\text { Subcontinent to } \\
\text { S. China, W. } \\
\text { Malaysia. }\end{array}$ & No \\
\hline $\begin{array}{l}\text { Portulaca oleracea } \mathrm{L} . \\
\text { [Portulacaceae]; LG-123 }\end{array}$ & Gubor-Oying & $\mathrm{F}$ & Whole plants edible & Australia & No \\
\hline $\begin{array}{l}\text { Gonostegia hirta (Blume ex } \\
\text { Hassk.) Miq. [Urticaceae]; MT- } \\
920\end{array}$ & Oyik & $\mathrm{F} ; \mathrm{M}$ & $\begin{array}{l}\text { Tender shoots edible; } \\
\text { leaf-paste used on cuts } \\
\text { and wound }\end{array}$ & $\begin{array}{l}\text { Trop. \& subtrop. } \\
\text { Asia to Australia }\end{array}$ & Yes \\
\hline $\begin{array}{l}\text { Pouzolzia zeylenica (L.) Benn. } \\
\text { [Urticaceae]; MT- } 921\end{array}$ & Oyik & $\mathrm{M}$ & $\begin{array}{l}\text { Leaf paste used on cuts } \\
\text { and wounds }\end{array}$ & $\begin{array}{l}\text { Trop. \& subtrop. } \\
\text { Asia. }\end{array}$ & No \\
\hline $\begin{array}{l}\text { Ricinus communis L. } \\
\text { [Euphorbiaceae]; LG-121 }\end{array}$ & $\begin{array}{l}\text { Gopo-Golo/ } \\
\text { Perok aki/Aki } \\
\text { rongmik }\end{array}$ & $\mathrm{M}$ & $\begin{array}{l}\text { Leaves warm on fire } \\
\text { placed paining joints and } \\
\text { muscle. Petiole bark used } \\
\text { as bandage to cure } \\
\text { fractured bone of chicks }\end{array}$ & N-E Africa & No \\
\hline $\begin{array}{l}\text { Rorippa dubia (Pers.) H.Hara } \\
\text { [Brassicaceae]; MT-918 }\end{array}$ & $\begin{array}{l}\text { Orgyam, sitong } \\
\text { pettu }\end{array}$ & $\mathrm{F}$ & Leaves edible & $\begin{array}{l}\text { Indian } \\
\text { subcontinent, } S \\
\text { China, Malay } \\
\text { peninsula }\end{array}$ & No \\
\hline $\begin{array}{l}\text { Rubia manjith } \text { Roxb. ex Fleming } \\
\text { [Rubiaceae]; MT- } 938\end{array}$ & Taman & $\mathrm{O}$ & $\begin{array}{l}\text { Roots boiled with cotton } \\
\text { (for thread) turns red }\end{array}$ & $\begin{array}{l}\text { Africa to trop. } \\
\text { Asia, China, } \\
\text { Japan, Australia }\end{array}$ & No \\
\hline $\begin{array}{l}\text { Rubus ellipticus Sm. [Rosaceae]; } \\
\text { MT-939 }\end{array}$ & $\begin{array}{l}\text { Tangkin / } \\
\text { Pakkom Tayin/ } \\
\text { Pakkom tasing }\end{array}$ & $\mathrm{F}$ & Sweet ripe fruits edible & $\begin{array}{l}\text { China, Nepal, } \\
\text { Indian } \\
\text { subcontinent, } \\
\text { Indochina, } \\
\text { Philippines }\end{array}$ & No \\
\hline $\begin{array}{l}\text { Rumex maritimus L. } \\
\text { [Polygonaceae]; MT-916 }\end{array}$ & Okung & $\mathrm{F}$ & Young leaves edible & Ireland & Yes \\
\hline $\begin{array}{l}\text { Saccharum arundinaceum Retz. } \\
\text { [Poaceae]; GM-141 }\end{array}$ & Tapii & $\mathrm{RBC}$ & $\begin{array}{l}\text { Believed that it is the } \\
\text { elder brother of Carex sp. } \\
\text { and both are use in rituals } \\
\text { during funeral }\end{array}$ & India & No \\
\hline $\begin{array}{l}\text { Saccharum spontaneum L. } \\
\text { [Poaceae]; MT-928 }\end{array}$ & $\begin{array}{l}\text { Piko-Pimur/ } \\
\text { Aasi-Pimur }\end{array}$ & $\mathrm{F}$ & $\begin{array}{l}\text { Roasted young } \\
\text { inflorescences edible }\end{array}$ & $\begin{array}{l}\text { Sicilia, Africa, } \\
\text { Asia to N. \& } \\
\text { NE. Australia. }\end{array}$ & No \\
\hline $\begin{array}{l}\text { Scoparia dulcis L. } \\
\text { [Plantaginaceae]; GM- } 147\end{array}$ & Yongin & $\mathrm{M}$ & $\begin{array}{l}\text { Taken orally against } \\
\text { rabies }\end{array}$ & C \& S America & No \\
\hline $\begin{array}{l}\text { Senna alata (L.) Roxb. } \\
\text { [Fabaceae]; LG- } 109\end{array}$ & Donyi Sori & $\mathrm{M}$ & $\begin{array}{l}\text { Leaf-paste used as } \\
\text { antimicrobial medicine } \\
\text { for old wounds }\end{array}$ & $\begin{array}{l}\text { N-S \& C } \\
\text { America }\end{array}$ & No \\
\hline $\begin{array}{l}\text { Senna tora }(\text { L.) Roxb. } \\
\text { [Fabaceae]; LG-110 }\end{array}$ & Donyi Sori & M & $\begin{array}{l}\text { Leaf-paste used against } \\
\text { Ring worm }\end{array}$ & C America & No \\
\hline $\begin{array}{l}\text { Sida acuta } \text { Burm.f. [Malvaceae]; } \\
\text { LG-128 }\end{array}$ & Jaru Ane & $\mathrm{O}$ & $\begin{array}{l}\text { Dried whole plants used } \\
\text { as hard broom }\end{array}$ & C America & No \\
\hline $\begin{array}{l}\text { Solanum americanum Mill. } \\
\text { [Solanaceae]; MT-914 }\end{array}$ & $\begin{array}{l}\text { Okomamang/Ma } \\
\text { ali/Yanga }\end{array}$ & $\mathrm{F}$ & $\begin{array}{l}\text { Tender shoots taken as } \\
\text { vegetable }\end{array}$ & \begin{tabular}{l|} 
America, \\
Melanesia, New \\
Guinea, Australia
\end{tabular} & Yes \\
\hline $\begin{array}{l}\text { Solanum torvum Sw. } \\
\text { [Solanaceae]; MT-905 }\end{array}$ & $\begin{array}{l}\text { Migom kopi, } \\
\text { Kodu/Taleng } \\
\text { koee }\end{array}$ & $\mathrm{F}$ & $\begin{array}{l}\text { Young fruits tastes bitter, } \\
\text { made into chutney }\end{array}$ & $\begin{array}{l}\text { Florida, S } \\
\text { Alabama, Brazil, } \\
\text { Mexico }\end{array}$ & Yes \\
\hline $\begin{array}{l}\text { Solanum viarum Dunal } \\
\text { [Solanaceae]; MT-924 }\end{array}$ & Peeli-Taang & $\mathrm{M}$ & $\begin{array}{l}\text { Warmed fruits used in } \\
\text { toothache }\end{array}$ & $\begin{array}{l}\text { Brazil, } \\
\text { Argentina }\end{array}$ & No \\
\hline $\begin{array}{l}\text { Solanum villosum Mill. } \\
\text { [Solanaceae]; MT-913 }\end{array}$ & $\begin{array}{l}\text { Okomamang/Ma } \\
\text { ali/ koieer }\end{array}$ & $\mathrm{F}$ & $\begin{array}{l}\text { Tender shoots taken as } \\
\text { vegetable }\end{array}$ & $\begin{array}{l}\text { Europe, W Asia, } \\
\text { N Africa, N } \\
\text { America }\end{array}$ & No \\
\hline $\begin{array}{l}\text { Sonchus oleraceus L. } \\
\text { [Asteraceae]; MT-911 }\end{array}$ & Ogon & $\mathrm{F}$ & $\begin{array}{l}\text { Tender leaves eaten as } \\
\text { vegetable }\end{array}$ & Europe, W Asia & No \\
\hline
\end{tabular}


Momang Taram et al.

\begin{tabular}{|c|c|c|c|c|c|}
\hline $\begin{array}{l}\text { Botanical name [Family]; } \\
\text { Voucher specimen }\end{array}$ & Adi Names & T U & Mode of use & $\begin{array}{l}\text { Endemism } \\
\text { range }\end{array}$ & $\begin{array}{l}\text { Marke- } \\
\text { tability }\end{array}$ \\
\hline $\begin{array}{l}\text { Spermacoce alata Aubl. } \\
\text { [Rubiaceae]; LG-113 }\end{array}$ & $\begin{array}{l}\text { Eeing / Tagin } \\
\text { mikki }\end{array}$ & FD & $\begin{array}{l}\text { Whole plant used as pig- } \\
\text { fodder }\end{array}$ & $\begin{array}{l}\text { S Mexico, C } \\
\text { America, } \\
\text { Caribbean }\end{array}$ & No \\
\hline $\begin{array}{l}\text { Spermacoce ocymoides Burm.f. } \\
\text { [Rubiaceae]; LG-112 }\end{array}$ & Eeing & FD & $\begin{array}{l}\text { Whole plant used as pig- } \\
\text { fodder }\end{array}$ & $\begin{array}{l}\text { Trop. to SW } \\
\text { Pacific }\end{array}$ & No \\
\hline $\begin{array}{l}\text { Spilanthes acmella }(\mathrm{L} .) \mathrm{L} . \\
\text { [Asteraceae]; MT-903 }\end{array}$ & $\begin{array}{l}\text { Marshang Haali, } \\
\text { Aying Marshang }\end{array}$ & $\mathrm{F}$ & Leaves edible & $\begin{array}{l}\text { Trop. \& S } \\
\text { countries mainly } \\
\text { India, S America }\end{array}$ & Yes \\
\hline $\begin{array}{l}\text { Stellaria media (L.) Vill. } \\
\text { [Caryophyllaceae]; LG-125 }\end{array}$ & Hosir Oying & $\mathrm{F}$ & Whole plants edible & Europe, Africa & Yes \\
\hline $\begin{array}{l}\text { Themeda villosa (Lam.) } \\
\text { A.Camas [Poaceae]; GM-144 }\end{array}$ & Tase & $\mathrm{O}$ & $\begin{array}{l}\text { Dried leaves use for } \\
\text { roofing }\end{array}$ & $\begin{array}{l}\text { Trop. \& subtrop. } \\
\text { Asia }\end{array}$ & No \\
\hline $\begin{array}{l}\text { Thysanolaena latifolia (Roxb. } \\
\text { ex Hornem.) Honda [Poaceae]; } \\
\text { LG-135 }\end{array}$ & $\begin{array}{l}\text { Kanggam/Jaru } \\
\text { Ane/Kamgang }\end{array}$ & $\begin{array}{l}\mathrm{RBC} ; \\
\mathrm{O}\end{array}$ & $\begin{array}{l}\text { Believe that in course of } \\
\text { evolution the tail of } B o s \\
\text { frontalis was originated } \\
\text { from plant inflorescence; } \\
\text { mature inflorescence use } \\
\text { as soft broom }\end{array}$ & $\begin{array}{l}\text { Trop. \& subtrop. } \\
\text { Asia }\end{array}$ & Yes \\
\hline $\begin{array}{l}\text { Urtica ardens Link [Urticaceae]; } \\
\text { LG-130 }\end{array}$ & $\begin{array}{l}\text { Jimang/Matpe } \\
\text { Pereng }\end{array}$ & $\mathrm{M}$ & $\begin{array}{l}\text { Infected wounds of Bos } \\
\text { frontalis is beaten with } \\
\text { nettle leaf to kill the } \\
\text { infectious organisms }\end{array}$ & $\begin{array}{l}\text { Himalayas to } \\
\text { SE Tibet. }\end{array}$ & No \\
\hline $\begin{array}{l}\text { Urtica dioca L. [Urticaceae]; } \\
\text { MT-904 }\end{array}$ & $\begin{array}{l}\text { Matpe pereng/ } \\
\text { Peji pamang }\end{array}$ & $\mathrm{M}$ & As in $U$. ardens & $\begin{array}{l}\text { Europe, temp. } \\
\text { Asia, W-N Africa }\end{array}$ & No \\
\hline $\begin{array}{l}\text { Viola betonicifolia } \mathrm{Sm} . \\
\text { [Violaceae]; LG-133 }\end{array}$ & Jortung / Japjor & $\mathrm{F}$ & Whole plants edible & $\begin{array}{l}\text { S Asia, E } \\
\text { Australia, } \\
\text { Tasmania }\end{array}$ & No \\
\hline $\begin{array}{l}\text { Viola pilosa Blume [Violaceae]; } \\
\text { LG-132 }\end{array}$ & $\begin{array}{l}\text { Jorsing/ Japjor } \\
\text { Peaak sungar }\end{array}$ & $\mathrm{F}$ & Whole plants edible & $\begin{array}{l}\text { Trop. \& temp. } \\
\text { Asia }\end{array}$ & No \\
\hline $\begin{array}{l}\text { Youngia japonica (L.) DC. } \\
\text { [Asteraceae]; MT-931 }\end{array}$ & $\begin{array}{l}\text { Rungdum/ } \\
\text { Rumdum/Rukjub } \\
\text { mikki }\end{array}$ & $\mathrm{O}$ & $\begin{array}{l}\text { Dried leaves are smoked } \\
\text { as substitute to tobacco }\end{array}$ & E Asia & No \\
\hline
\end{tabular}

\section{Ethnomedicinal uses}

Of the total 87 weed species reported, $27 \%$ of them are being used in traditional ethnomedicinal practices of the Adi community for the treatment of different ailments such as Gastrointestinal disorder, cuts and wound, burns and inflammation, malaria, toothache, jaundice, files, sinus, crack sole, ringworm, sprain and rabies. Majority of the total species reported are used for treatment of Gastro-intestinal disorder, cuts and wound, burns and inflammation and malaria, whereas few species such as Urtica dioica and Urtica parviflora were found to be used in ethno-veterinary medicinal practices for the treatment of infected wound of their livestock population.

\section{Animal fodder and forage}

Domestication of animal is the primary occupation in the rural localities to sustain the economy and human livelihood. Present investigations have revealed a total of 9 weedy species exclusively used by the rural farmers as livestock fodder for the ruminant cattle, such as Mithun, Cow, and Goat. Some of the species are luxuriantly growing in the wild without any agronomic care and are non-toxic, but rather nutritious which are mostly foraged by the ruminant animals.

\section{Weed plant in traditional rituals}

Adi community being the followers of indigenous Donyi-Polo religious group uses several plant species while worshiping Gods and Goddesses like Kine Nane, Doying Bote, Gumin 
Soyin, Pedong Nane, and Dadi bote, which is performed with invocation of hymns as per their existing ritual traditions. Present investigation have revealed a total of 8 weedy species which are widely used in traditional rituals, and also associated with local folk belief systems. Weeds species namely, Thysanolaena latifolia and Chenopodium giganteum are mentioned in folklores since time immemorial which are deeply associated with indigenous faith and belief systems of the Adi community.

\section{Commercially important weeds}

A good share of $20 \%$ of the recorded 87 species are commercially important, which are frequently harvested and sold in markets as food and medicinal items. These sellers are mostly the marginal farmers, mainly women to sustain their livelihood. These species are also extensively harvested in some places for vermicomposting to produce organic manure in Kitchen Gardens for ensuring organic crop production.

\section{CONCLUSION}

It is concluded that Adi people of Siang district are rich in traditional knowledge and local skills related to wise use of weed flora available in their bio cultural landscape. The weed flora luxuriantly growing almost everywhere in Siang Districts of Arunachal Pradesh are being converted into economically productive and livelihood based support items by the local residents by employing them in diverse uses such as food, human medicine, veterinary medicine, organic manure, rituals and belief systems.

\section{Acknowledgements}

The authors are thankful the DBT New Delhi for funding under Twinning programme and BRNS DAE BARC Trombay for fund support to RGU. They are also thankful SERB DST New Delhi and GBPIHESD under IERP programme for fund support to NIT, Arunachal Pradesh for conducting present research. All the authors are thankful to the local informants of Adi community residing in 8 villages of Upper Siang, Siang and East Siang districts of Arunachal Pradesh for sharing their valuable ethnobotanical knowledge system during field visits.

\section{LITERATURE CITED}

Baker, H.G. 1965. Characteristics and modes of origin of weeds. In: Baker, H.G., Stebbins, G.L. (eds.), The Genetics of Colonizing Species. Academic Press, New York. Pp. $147-172$.

Chowdhery, H.J.; Giri, G.S. \& Pramanik, A. 2009. Materials for the Flora of Arunachal Pradesh. Vol. III. Botanical Survey of India, Calcutta.

Dhole, J.A; Dhole, N.A. \& Bodke S.S. 2009. Ethnobotanical studies of some weeds of crop fields of Marathwada region, India. Ethnobot. Leafl. 13(1): 1443 - 1452.

Giri, G.S.; Pramanik, A. \& Chowdhery, H.J. 2008. Materials for the Flora of Arunachal Pradesh. Vol. II. Botanical Survey of India, Kolkata.

Hajra, P.K.; Verma, D.M. \& Giri, G.S. 1996. Materials for the Flora of Arunachal Pradesh. Vol. I. Botanical Survey of India, Calcutta.

Hooker, J.D. 1872 - 1897. The flora of British India, Vols. 1 - 7. L.Reeve \& Co Ltd, Ashford, Kent. London

Jain, S.K. \& Rao, R.R. 1977. A Handbook of Field and Herbarium Methods. Today and Tomorrow Printers and Publishers, New Delhi. 
Maroyi, A. 2013. Use of weeds as Traditional vegetables in Shurugwi District, Zimbawe. Journal of Ethnobio. Ethnomed., 60(9): 1 - 10.

Martin, G.J. 2008. Ethnobotany: A Methods Manual, People and Plants Conservation Series. Earthscan, UK and USA. Pp. $10-160$.

Neogi, B; Prasad, M.N.V. \& Rao, R.R. 1989. Ethnobotany of some weeds of Khasi and Garo Hills, Meghalaya, Northeastern India. Econ. Bot. 43(4): 471 - 479.

Rahman, A.H.M.M. 2013. A Checklist of Common Angiosperm Weeds of Rajshahi District, Bangladesh. Intn. Invent. J. Agri. Soil Sci. 1(1): $1-6$.

Rahman, A.H.M.M; Islam, A.K.M.R \& Rahman, M.M. 2011. The Family Asteraceae of Rajshahi Division, Bangladesh. VDM Verlag Dr. Muller Publishing House Ltd., Germany.

Rao, A.N. \& Chauhan, B.S. 2015. Weeds and weed management in India - A Review. Chapter 4. In: Rao, V.S.; Yaduraju, N.T.; Chandrasena, N.R.; Hassan, G. \& Sharma, A.R. (eds.), Weed Science in the Asian-Pacific Region. An AsianPacific Weed Sci. Soc. Public. Indian Soc. Weed Sci., Jabalpur, India. ISBN 13: 978-81-931978-0-6. Pp. $87-118$.

Tag, H; Murtem, G; Das, A.K. \& Singh, R.K. 2008. Diversity distribution of ethnobotanical plants used by Adi tribe of East Siang district of Arunachal Pradesh, India. Pleione 2(1): $123-136$.

Zimdahl, R.L. 1992. Fundamentals of Weed Science, $2^{\text {nd }}$ edn., Academic Press, San Diego, CA.

http://www.theplantlist.org

http://www.plantsoftheworldonline.org/ 